\title{
Tiny wasp helps protect eucalypts from eucalyptus longhorned borer
}

\author{
Lawrence M. Hanks $\square$ Timothy D. Paine $\square$ Jocelyn G. Millar
}

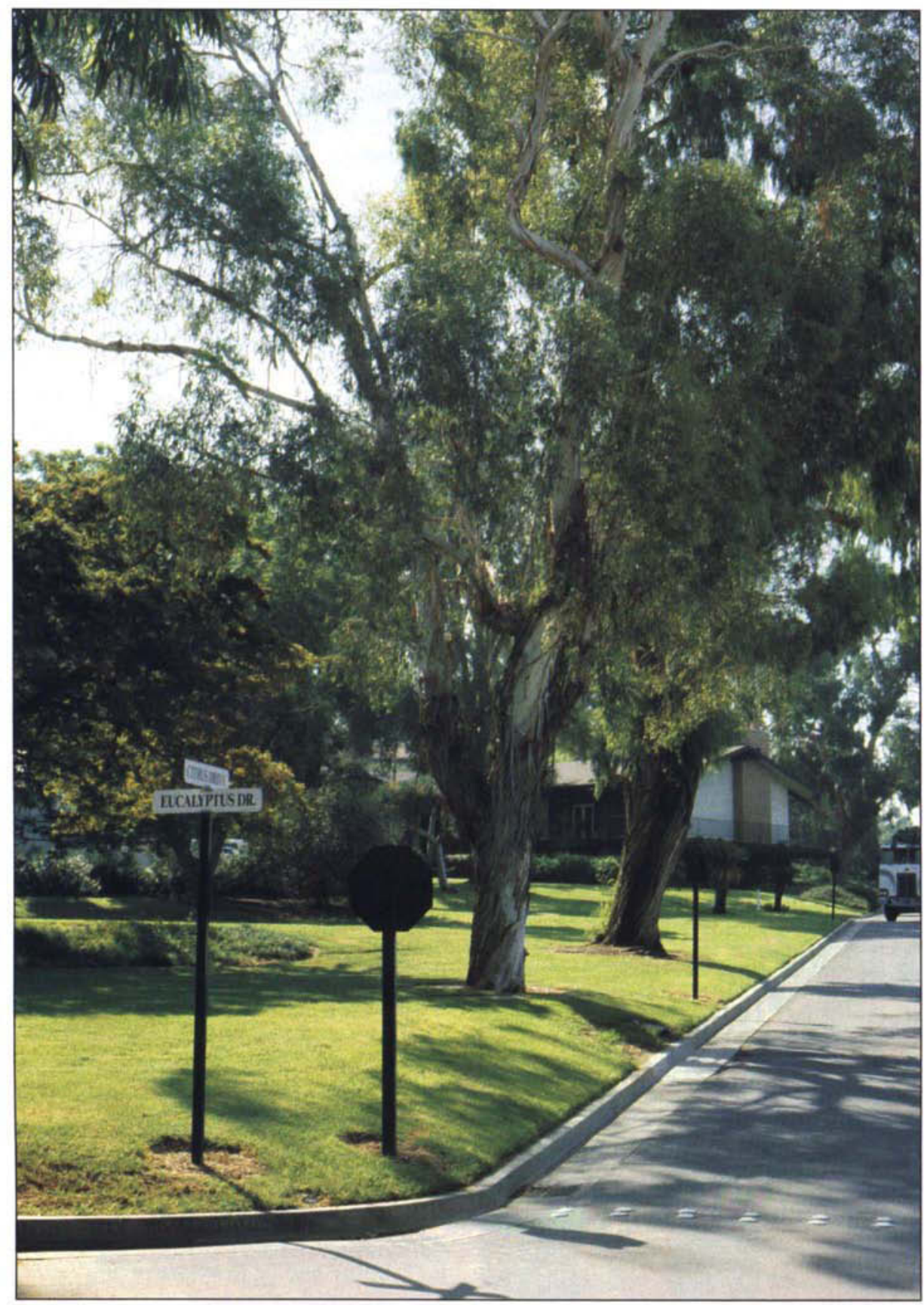

Damaged eucalyptus tree on suburban street. Without a natural enemy to keep it in check, the eucalyptus longhorned borer has killed thousands of eucalypts throughout the state.
The eucalyptus longhorned borer (ELB) was first identified in California in 1984 and has since killed thousands of eucalyptus trees. Now firmly established throughout Southern California, the borer continues to spread northward. A search for its natural enemies in Australia yielded a tiny wasp, Avetianella longoi, that parasitizes ELB eggs. The wasp was imported, released and is now established in San Diego and Riverside counties. Results of releases in other areas are pending. A. longoi propagates quickly, disperses rapidly across wide areas, and is highly efficient at finding and parasitizing borer eggs.

In 1984, a eucalyptus tree in Orange County was discovered to be infested with the larvae of an insect species new to this continent, the eucalyptus longhorned borer (ELB) (California Agriculture July-August 1986). The appearance of this large beetle in California was alarming because of its reputation as a killer of eucalypts in most countries where these trees grow. ELB lay their eggs under the loose bark of the trunk and branches, and the larvae penetrate the bark to feed in the cambium. By consuming these critical tissues, which conduct water and nutrients, the larvae can destroy a tree in just a few weeks. ELB is an economically significant pest, affecting nearly every region in the world where eucalypts have been planted including Portugal, Spain, Italy, Israel, Egypt, Tunisia and South Africa.

ELB threatens the numerous, important roles of eucalypts in the Cali- 


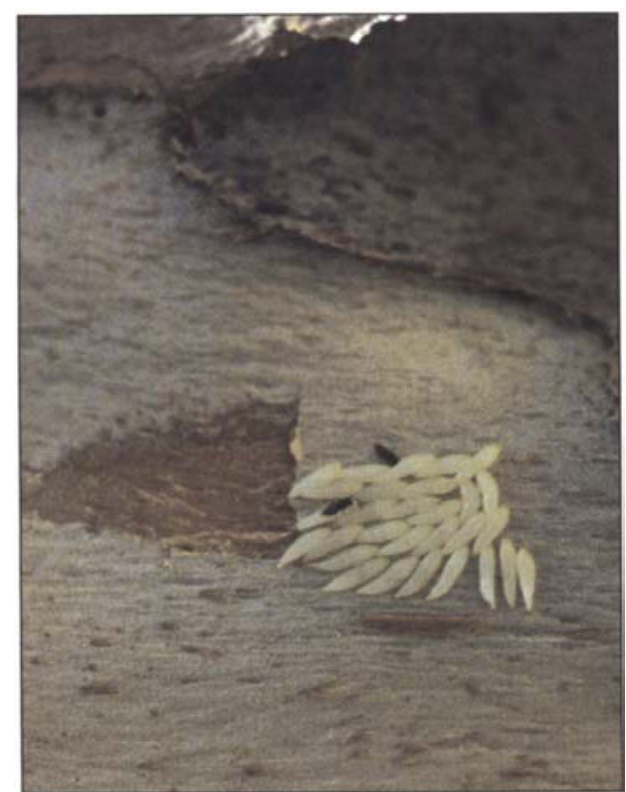

A Avetianella longoi are attracted to egg batches of the eucalyptus longhorned borer.

fornia landscape. First brought to the state in 1856, eucalypts rapidly gained popularity as shelter, shade and ornamental trees because they thrived without care even on marginal sites. In Southern California, eucalypts are abundant in places originally treeless, and, in some cases, comprise almost the entire urban forest - from intensively managed parks and golf courses to entirely unmanaged stands on rangeland and undeveloped land. Eucalypts are locally abundant in the central and northern parts of the state as well, where they serve as roadside and windbreak trees. They are also grown for use as biomass, pulpwood and fuelwood.

The eucalyptus pest has lived up to its bad reputation in the decade since its colonization of California. On the UC San Diego campus and in the community of Rancho Santa Fe (San Diego County) alone, thousands of eucalypts have succumbed to attack. The beetle continues to spread northward, causing equally severe tree kills in Santa Barbara and the San Francisco Bay Area, with new infestations reported in Fresno, Sacramento and Glenn counties.

Although ELB is effectively destroying eucalypts in California, it rarely achieves pest status in its homeland, Australia. There, eucalypts growing in their natural environments

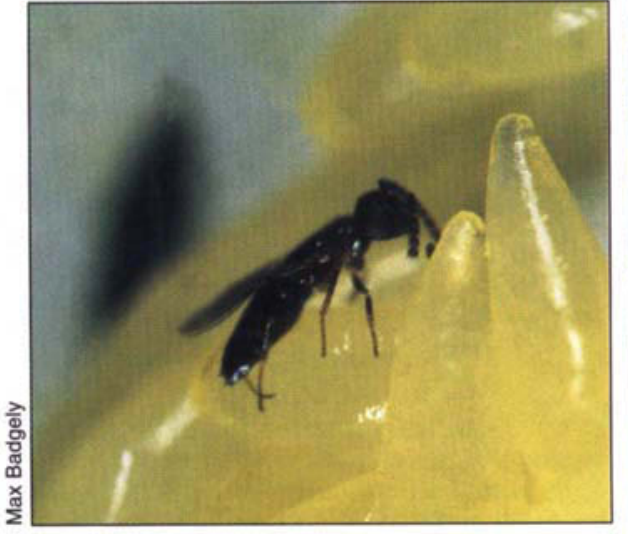

A Avetianella longoi females insert their eggs into eggs of the borer.

Larvae of $A$. longoi develop within the eggs of the eucalyptus longhorned borer. Dark eggs contain adult parasites almost ready to emerge.

appear to be more resistant to beetle attack, and the beetle itself falls prey to many natural enemies. No such ecological checks and balances exist in California, where eucalypts experience subtle stresses, particularly water deficit, that may compromise their resistance; consequently, ELB populations have exploded in the absence of natural enemies.

\section{ELB parasite found}

Losses to the beetle can be mitigated by bolstering tree resistance through appropriate pruning and irrigation practices, and by planting eucalyptus species that have higher innate resistance (see California Agriculture January-February 1995). To reduce further losses of eucalypts, we have imported a natural enemy of the borer from Australia for release in California. This stingless wasp is a highly specialized parasite of eucalyptus borers that poses no threat to native insect species, nor can it sting people.

In 1992, Dr. Qiao Wang of La Trobe University in Melbourne, Australia, working in collaboration with UC Riverside entomologists, discovered ELB eggs that had been parasitized. From these parasitized eggs emerged tiny. wasps, no larger than grains of black pepper, that were subsequently identified as Avetianella longoi Siscaro. After determining that the wasp was highly

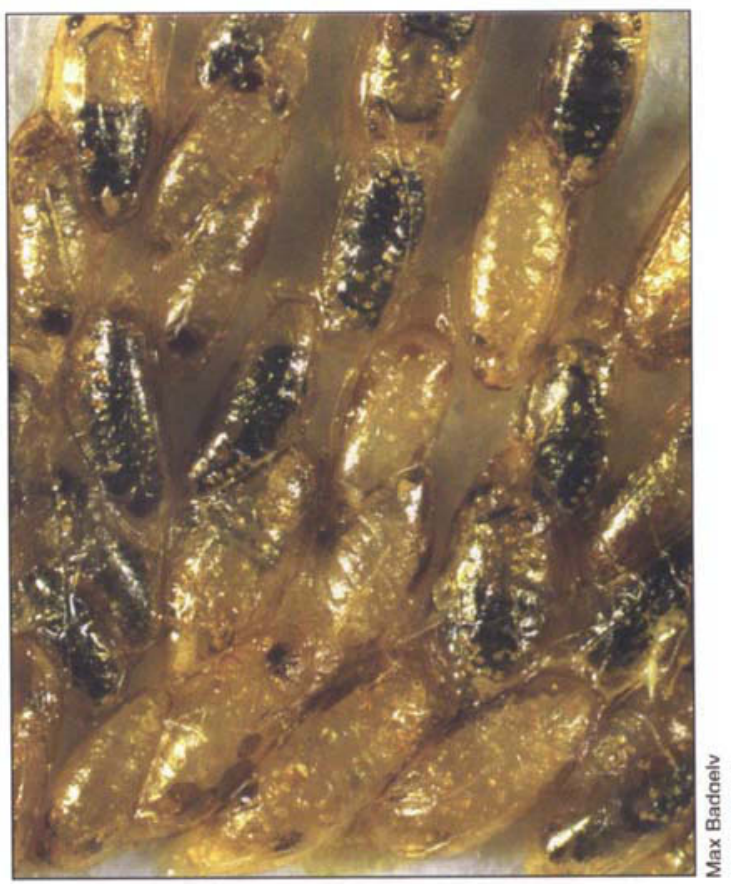

host-specific, we imported it into California as a biological control agent of the borer.

Adult $A$. longoi have a flattened body form that allows them to squeeze under loose bark to reach ELB eggs. Female wasps insert their eggs into the borer eggs, and the developing wasp larvae consume the contents of the borer egg. Each larva completes development within a single ELB egg in about 16 days, then the adult wasp emerges by chewing a hole in the eggshell. As many as five wasps complete development in a single borer egg, and female A. longoi can parasitize about 200 eggs during their life span, which averages 26 days. This high reproductive capacity and short generation time suggest that $A$. longoi can quickly build its population in response to an abundance of ELB eggs.

\section{Wasp releases}

During summer 1993, we released more than 53,000 A. longoi in San Diego and Rancho Santa Fe (San Diego County), the city of Riverside (Riverside County), Fontana (San Bernardino County), Will Rogers State Park (Los Angeles County), and on the Stanford University campus (Santa Clara County). By fall, ELB eggs parasitized by the wasp were found on eucalyptus trees and fallen branches at sites distant from all the release points. For ex- 
ample, we released wasps on the UC San Diego campus and later found two egg batches (44 eggs total) that were completely parasitized about 9.3 miles $(15 \mathrm{~km})$ away at the San Diego Zoo. In the field, we observed as many as four adult $A$. longoi on a single batch of borer eggs. These wasps did not appear to fly readily, but took short, hopping flights. Nevertheless, the wasps spread at a remarkable rate, especially given the seemingly inhospitable and mostly urbanized terrain they had to traverse. Strong wasp dispersal ability would be necessary for control of ELB because the beetles can fly long distances in search of their eucalyptus host trees.

Although populations of $A$. longoi were spreading from all release sites, they still had to survive the winter. Because ELB are not active during winter, fresh host eggs are not available to the wasps for several months. A. longoi is known to pass the winter as pupae inside the shells of the borer eggs. If the adult wasps were to become active at the wrong time, however, they would not find host eggs, and wasp populations would go extinct. In the spring of 1994, adult $A$. longoi reappeared on eucalyptus logs at the Riverside site, giving the first confirmation that they had survived the winter and were permanently established. We also found parasitized eggs in San Diego and Rancho Santa Fe, but not at Will Rogers State Park or Stanford University. No ELB eggs could be found at the Fontana site to confirm that the wasp was present, and trap logs put out to encourage egg laying by the beetles had been pilfered.

We continued to release $A$. longoi through 1994 and 1995 at the Stanford site where the wasp had failed to establish, and at new sites at Chollas Lake (San Diego County), in Irvine (Orange County), Santa Barbara (Santa Barbara County), Long Beach and Santa Clarita (Los Angeles County) and Fresno (Fresno County). More than $121,000 \mathrm{~A}$. longoi were released at these seven sites during 1994 and 1995 , and we hope to confirm establishment in 1996.

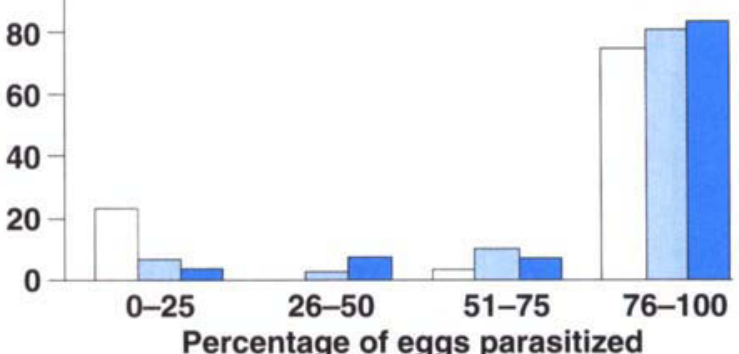

With fewer borer larvae attempting to feed on the cambium, the trees will have a greater chance of resisting and surviving attack.

The use of $A$. longoi as a biological control agent of ELB is also being explored in other regions of the world. This wasp was apparently accidentally introduced into the Mediterra-

Fig. 1. Frequency distribution of the percentage of eucalyptus longhorned borer eggs within a batch that were parasitized by Avetianella longoi. Egg batches were collected in San Diego and Riverside counties in summer and fall of 1993 ( $N=$ 40 batches), 1994 ( $N=97$ batches), and 1995 ( $N=63)$.

\section{A. longoi is efficient parasite}

A. longoi showed promise as a biological control agent by surviving the winter at most release sites, and by effectively locating ELB eggs in varied habitats and on numerous eucalyptus species. Additionally, it proved a worthy adversary of ELB because of the high proportion of eggs it killed.

We collected 40 ELB egg batches at the San Diego, Rancho Santa Fe and Riverside sites in summer and fall of 1993, 97 batches in 1994, and 63 batches in 1995. Most of the egg batches in 1993 were more than 75\% parasitized (fig. 1), and $71 \%$ of all eggs collected were parasitized. Even fewer egg batches escaped parasitism in 1994 and 1995 , even though we had not released parasites at these sites since the previous year; $81 \%$ and $84 \%$ of batches collected in those years were more than $75 \%$ parasitized (fig. 1), with $91 \%$ and $88 \%$ of all eggs parasitized. The decline from 1993 to 1995 in the percentage of egg batches that were less than $25 \%$ parasitized (fig. 1 ), and the corresponding increase in the number of highly parasitized batches, are statistically significant $\left(x^{2}=17.3\right.$, $\mathrm{df}=6, P<.0 \mathrm{l})$.

The high rates at which $A$. longoi parasitized ELB eggs, coupled with its strong powers of dispersal and efficient location of host eggs, suggest that this wasp is likely to have an important impact on the ELB problem in California. The wasp sharply curtails the number of ELB larvae that survive to penetrate the bark of eucalypts. nean area, where it now parasitizes high percentages of ELB eggs. We have also supplied $A$. longoi to researchers for rearing and release in the Republic of South Africa, where the wasp has already spread more than 80 miles $(130 \mathrm{~km})$ in only 2 years. There the parasite also crossed 8.7 miles (14 km) of open sea, reaching an island where eucalypts were being attacked by ELB.

\section{Other natural enemies}

We will continue to release $A$. longoi in California in areas where it has not yet become established. In addition, we are also releasing three highly specialized braconid wasps that parasitize ELB larvae feeding under the bark of eucalyptus. One species, Syngaster lepidus Brullé, already shows evidence of reproducing in the field; we have found hundreds of their emergence holes. By establishing permanent populations of these natural enemies in California, we hope to tip the ecological balance in favor of the eucalypts, which have become such an important feature of the California landscape.

\section{L.M. Hanks is Assistant Research Ento-} mologist, T.D. Paine and J.G. Millar are Associate Professors, Department of Entomology, UC Riverside.

The authors wish to thank the Rancho Santa Fe Association, Elvenia Slosson Fund for Ornamental Horticulture, California Department of Forestry and Fire Protection, California Department of Transportation, UC Statezvide Integrated Pest Management Program and the California Association of Nurserymen for their support. We also thank J. Gould, F. Ameeri, A. Rust and S. Gilbert for their contributions and assistance. 